\title{
Una propuesta metodológica para trabajar la violencia de género con adolescentes a través de las canciones
}

A methodological proposal to work on gender violence with teenagers through songs

\author{
Jaime Hormigos Ruiz \\ jaime.hormigos@urjc.es \\ Departamento de Ciencias de la Comunicación y Sociología \\ Universidad Rey Juan Carlos \\ Madrid, España \\ ORCID: 0000-0002-9728-2256
}

doi: 10.7203/LEEME.46.16999

\section{Resumen}

En nuestra sociedad las cifras de agresiones machistas aumentan entre la población adolescente a pesar de los esfuerzos realizados desde las administraciones para erradicar la violencia de género. Este trabajo de investigación centra su análisis en el discurso musical que rodea al adolescente para poder establecer una relación entre la presencia de mensajes machistas en los contenidos culturales y la normalización de estos comportamientos en sus pautas de interacción social. Para alcanzar nuestro objetivo, en primer lugar, desarrollamos una metodología cuantitativa basada en el análisis de contenido de una muestra de 350 canciones cuyas letras tienen relación con esta temática. Los resultados muestran que a pesar de que los mensajes en contra de la violencia de género son más habituales en las canciones analizadas, los contenidos que potencian la violencia de género son los más distribuidos por las industrias culturales y, por tanto, los que más éxito tienen entre los jóvenes. En segundo lugar, proponemos una metodología de trabajo que nos permite utilizar en el aula el poder educativo de la música para socializar al adolescente en valores que le ayuden a identificar y combatir la violencia de género.

Palabras clave: Educación; violencia de género; adolescentes; canciones de música popular.

\begin{abstract}
In our society the numbers of sexist aggressions increase among teenagers. This research proposes an analysis of the adolescents' musical discourse that establishes a relationship between the presence of sexist content in these cultural contents and the normalization of these behaviors in their social interaction patterns. First, the work proposes a quantitative methodology based on the content analysis of a sample of 350 songs whose lyrics are related to gender violence. The results show that although the messages against gender violence are more common in the songs analyzed, the contents that promote gender violence are the most distributed by the cultural industries and, therefore, the most successful among teenagers. Secondly, the paper proposes a work methodology that uses the educational power of music to socialize adolescents in values that help them identify and combat gender violence.
\end{abstract}

Key words: Education; gender violence; teenagers; popular music songs.

@ Jaime Hormigos Ruiz. The content of this article is the sole responsibility of the authors. The Revista Electrónica de LEEME and Universitat de València are not liable for any legal actions that may arise involving the article's content. Revista Electrónica de LEEME - Lista Electrónica Europea de Música en la Educaciónhttp://ojs.uv.es/index/php/LEEME/index ISSN: 1575-9563. Editores: Universidad de Valencia y Jesús Tejada. Visibilidad de esta revista: SCOPUS, Emerging Sources Citation Index (Clarivate), EBSCO, CINDOC (CSIC), Citefactor, COPAC, Dialnet, DICE (CSIC), DOAJ, e-revistas (CSIC), EBSCO Premier, ERIH+, Gale Cengage Learning, INRECS, IRESIE, LATINDEX, MIAR, OCLC Worldcat, RESH, REDIB, RILM Core Journals, SUDOC, ULRICHS. Esta revista es de acceso libre mediante licencia Creative Commons $4.0 \mathrm{CC}$ by. Política de archivado: etiqueta verde SHERPA-ROMEO. 


\section{Introducción}

En 2019 la violencia de género entre adolescentes creció de forma exponencial situándose en máximos históricos en España. Durante este periodo, el 14,8 \% de las denuncias por maltrato las realizan mujeres de menos de 18 años y el $18,7 \%$ de los denunciados por este tipo de violencia fueron también adolescentes (INE, 2020). La violencia se normaliza dentro del proceso de socialización cultural del joven hasta llegar a impregnar todos los subsistemas de su universo colectivo de manera subliminal (Prendes, García y Solano, 2020). Este tipo de violencia cultural se justifica a través del ámbito simbólico y se materializa en las formas de ocio, la moda, la música, el cine, los videojuegos o la publicidad y se utiliza, en cierto modo, para justificar o legitimar la violencia directa o la violencia estructural (García, Ruiz y Rebollo, 2016). Esta investigación analiza, en primer lugar, la presencia de la violencia de género en los productos musicales que utilizan los adolescentes. En segundo lugar, estudia cómo se trata este problema en los estilos musicales más distribuidos en la sociedad. Finalmente, propone una metodología educativa a partir del análisis de una muestra de canciones que denuncian la violencia de género empoderando el papel de la mujer.

\section{Revisión teórica}

En la sociedad actual los jóvenes identifican claramente los ejemplos más visibles de la violencia machista, pero son incapaces de reconocer signos de abuso en sus relaciones afectivas (Padrós, Aubert y Melgar, 2010). Ante esta perspectiva, es necesario establecer estudios que contribuyan a una resocialización preventiva del adolescente, que le ayuden a entender mejor normas y valores sociales que previenen los comportamientos y las actitudes violentas contra las mujeres y le enseñen comportamientos igualitarios y respetuosos (Valls, Puigvert y Duque, 2008). En este sentido, trabajar con las canciones como herramienta educativa puede ayudar ya que favorecen la creatividad y la comprensión de los aspectos esenciales de la vida del adolescente (Berrón, 2017). La música en el contexto social actual ha pasado de ser un elemento cultural unido a la transmisión de conocimientos y experiencias a ser un elemento de ocio y consumo más. Dentro del proceso de educación, se la relaciona más con la parte ociosa que con la parte formativa, ocupando en el aula una posición inferior a la que ocupa en la vida del individuo (Flores, 2007). En este sentido, es necesario potenciar el trabajo en el aula de los hábitos de escucha activa para revertir esta situación y favorecer una actitud más crítica frente a los contenidos culturales que rodean al adolescente (Kratus, 2017).

En España, en cifras generales, los hábitos de lectura entre los jóvenes han crecido en los últimos años. Ahora bien, el interés por la lectura cae a partir de los 15 años (FGEE, 2019), 
edad en la que la música comienza a ser más significativa en el universo del joven. De acuerdo con estos datos, se puede inferir que la adolescencia es la etapa en la que el individuo recibe más información a través de la música. En esta fase, los jóvenes utilizan las canciones como un potente elemento de comunicación a través del cual pueden construir una valoración sobre los problemas que suceden en su entorno (Branch, 2012). Por tanto, es en esta etapa donde la música se configura como una herramienta interesante para incorporar al proceso educativo.

La música no puede entenderse como algo ajeno al currículum formativo del adolescente. En el proceso de asimilación de la música a las pautas de ocio actuales, se están estableciendo cambios importantes en la forma de escucha y en la transmisión de valores a través de los textos de las canciones. Debido a la continua exposición a un discurso musical saturado, los jóvenes aprenden a desarrollar una escucha simple de las canciones desde la que interpretan su significado según modelos estandarizados muy influidos por las industrias culturales (Hennion, 2010). Así, la música se entiende más como un elemento de diversión que acompaña a la hora de realizar otras actividades. Este modelo de escucha no permite identificar de forma precisa los mensajes que llegan a través de las canciones, exponiendo al adolescente a los estilos musicales donde se distribuyen valores censurados por la sociedad (Karvelis, 2018). Trabajar en el aula los problemas sociales por medio de canciones capacita al adolescente para poder realizar una escucha más atenta de la música y favorece su actitud crítica.

La información que el alumnado adolescente recibe a través de las canciones que escucha juega con el factor temporal, comunica en el momento de ser recibida por el oyente, pero, además, por su estrecha relación con el mundo simbólico de la cultura, tiene la capacidad de transformar su significado con cada nueva audición (Hall, 2014). En este sentido, el discurso de la violencia de género en las canciones es una constante, unas veces cargando los temas de connotaciones negativas que potencian las desigualdades y otras, denunciando la situación. Este discurso, sobre todo en su parte más negativa, habitualmente pasa desapercibido en las primeras audiciones y solo se muestra cuando se presta una mayor atención al contenido de la letra (Hormigos, Gómez y Perelló, 2008). Esto sucede porque se dota de significado a la canción en función de los marcos personales de referencia que tienden a presentar como normales ciertas expresiones o valores que potencian la violencia de género. Por este motivo es tan importante trabajar la música en el proceso educativo como un hecho social que contribuya a crear identidades grupales que ayuden al adolescente a decodificar y a interpretar su significado (Kelly, 2002).

La adolescencia es una etapa fundamental para la formación de los gustos musicales. En esta etapa se aprende a escuchar nuevos sonidos y a percibir la vida a través de la música (Lorente, 2007). En este sentido, se responde mejor ante los diversos estímulos musicales en busca de aquellos sonidos que representan la forma en que la persona percibe la realidad. A medida que las personas crecen se identifican con otras preocupaciones distintas que no encuentran un reflejo directo en las canciones de moda y optan por seguir escuchando aquellas

@ Jaime Hormigos Ruiz. The content of this article is the sole responsibility of the authors. The Revista Electrónica de LEEME and Universitat de València are not liable for any legal actions that may arise involving the article's content. Revista Electrónica de LEEME - Lista Electrónica Europea de Música en la Educación-. http://ojs.uv.es/index/php/LEEME/index ISSN: 1575-9563. Editores: Universidad de Valencia y Jesús Tejada. Visibilidad de esta revista: SCOPUS, Emerging Sources Citation Index (Clarivate), EBSCO, CINDOC (CSIC), Citefactor, COPAC, Dialnet, DICE (CSIC), DOAJ, e-revistas (CSIC), EBSCO Premier, ERIH+, Gale Cengage Learning, INRECS, IRESIE, LATINDEX, MIAR, OCLC Worldcat, RESH, REDIB, RILM Core Journals, SUDOC, ULRICHS. Esta revista es de acceso libre mediante licencia Creative Commons 4.0 CC by. Política de archivado: etiqueta verde SHERPA-ROMEO. 


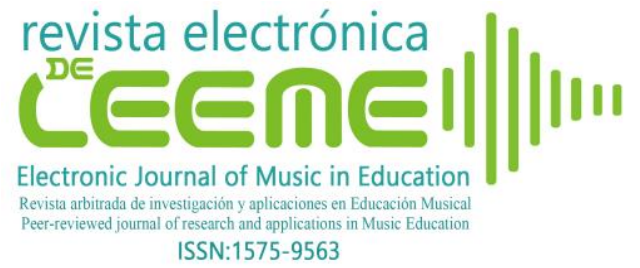

que más les marcaron durante la juventud, incluso cuando estas canciones transmitan unos valores que no encajan con la sociedad actual. Las distintas generaciones han crecido en una sociedad donde los roles del hombre y la mujer estaban claramente diferenciados y esto se ha reflejado de forma directa en las canciones que han ido escuchando (Padrós, Aubert y Melgar, 2010). Actualmente, aparece un doble problema; por una parte, la juventud se rodea de estilos musicales que fomentan la violencia de género como algo habitual y, por otra, se exponen a la música de sus progenitores, que en muchos casos justifica los celos, el maltratado físico y psicológico o la discriminación, creando un clima de cierta normalidad de la violencia de género (Herrera, Cremades y Lorenzo, 2010).

La distribución de la música en la sociedad afecta de forma significativa a la percepción que el adolescente tiene sobre el discurso musical que le rodea. Si una canción que fomenta la violencia de género suena constantemente en los medios, su mensaje se tenderá a relativizar y contribuirá a naturalizar ciertos comportamientos violentos (Gómez, Hormigos y Perelló, 2019). Por este motivo, es de vital importancia para conocer el problema estudiar el peso que tienen las canciones que tratan sobre la violencia de género, fomentándola o denunciándola, dentro de la sociedad actual.

\section{Método}

El objetivo general de este estudio es plantear una metodología de análisis de las canciones que permita enseñar al alumnado adolescente la importancia de aplicar la escucha activa sobre el discurso musical que le rodea. Esta escucha activa debe contribuir a desarrollar su actitud crítica frente a la aparición de valores que fomentan la violencia de género en los contenidos culturales. Para ello, es importante conocer la presencia de los discursos sobre la violencia de género en las canciones de música popular con el fin de identificar el material auditivo que se usará en la metodología de trabajo en el aula.

\subsection{Diseño}

El trabajo comienza desarrollando una investigación cuantitativa sobre una muestra de canciones de música popular que tratan en sus letras la violencia de género, fomentándola o criticándola. Este análisis musical se desarrolla a lo largo de cuatro décadas ya que la formación del oído musical del alumnado adolescente no tiene únicamente que ver con sus gustos musicales actuales, sino que la exposición constante a los estímulos musicales en el ámbito social también condiciona la recepción de valores a favor o en contra de la violencia. 


\subsection{Muestra}

En el marco de un muestreo estratégico intencional (Perelló-Oliver, 2009), se seleccionó una muestra de 350 canciones tras un análisis exhaustivo de las más escuchadas en España por año, según los criterios de ventas y distribución que recogen las dos principales agencias nacionales de gestión musical: Productores de Música de España (Promusicae, 2003-2019) y la Sociedad General de Autores y Editores (SGAE, 1999-2019). Estos datos se contrastaron con las estadísticas sobre las canciones más escuchadas por personas de 11 a 20 años que generan operadores como Spotify, Amazon, Boungiorno, Gran Vía Musical, Google Play, i-Tunes, Jetmultimedia, Movistar, Orange, Vodafone, Nokia, 7Digital y Zune (IFPI, 2006-2019).

La selección se basó en la escucha del listado de canciones más distribuidas, utilizando para la investigación aquellos temas cuya letra estuvieran en castellano porque transmiten de una forma más directa la percepción sobre la violencia de género en el alumnado adolescente y favorecen el proceso de escucha activa.

\subsection{Variables}

La muestra objeto de estudio se analizó a partir de las variables: 1. Estilo; 2. Fomento de la violencia de género; 3. Denuncia de la violencia de género; 4. Empoderamiento de la mujer; 5. Década y 6. Distribución. A partir de estas variables, se procedió a la codificación del conjunto de las canciones que integran la muestra en tres rondas sucesivas e independientes para garantizar la fiabilidad del proceso.

\section{Resultados}

En la muestra analizada, el $28 \%$ de las canciones que tratan la violencia de género lo hacen para fomentarla frente a un $72 \%$ que denuncia esta situación. Estos datos llevarían a pensar que el discurso que fomenta la violencia de género en las canciones tiene una presencia minoritaria frente al que denuncia este tipo de actitudes. Ahora bien, el dato relevante aparece cuando se analiza la distribución que tienen estas canciones en la sociedad como se muestra en la Tabla 1. 
Tabla 1. Canciones que fomentan o denuncian de la violencia de género por década y distribución masiva. En porcentajes $(\mathrm{n}=350)$

\begin{tabular}{c|cc|cc}
\hline Década & Fomenta & $\begin{array}{c}\text { Distribución } \\
\text { masiva }\end{array}$ & Denuncia & $\begin{array}{c}\text { Distribución } \\
\text { masiva }\end{array}$ \\
\hline Antes 1980 & 6,1 & 4,1 & 2,8 & 1,6 \\
\hline $1980-1989$ & 16,3 & 6,1 & 5,2 & 1,2 \\
\hline $1990-1999$ & 18,4 & 11,2 & 11,5 & 2,4 \\
\hline $2000-2009$ & 24,5 & 8,2 & 53,6 & 11,9 \\
\hline $2010-2018$ & 34,7 & 23,5 & 26,9 & 5,5 \\
\hline Total & 100 & 53,1 & 100 & 22,6 \\
\hline \multicolumn{5}{r}{ Fuente: elaboración propia }
\end{tabular}

El 53,1\% de todas las canciones de la muestra que fomentan la violencia de género se distribuyen masivamente, frente a una distribución de solo el 22,6 \% de todas las canciones que la denuncian. Esto provoca que los discursos violentos hacia la mujer estén más presentes en la vida diaria del adolescente a través de canciones de moda proyectadas constantemente en los medios de comunicación, plataformas musicales y redes sociales (Valls, Puigvert y Duque, 2008). Los datos son aún más significativos cuando se analiza la tendencia a lo largo de los años. Hasta la década 2000 - 2009 era habitual encontrar en las canciones más contenidos que fomentaban la violencia de género que contenidos que la denunciaban. La tendencia cambia hacia el año 2004 a medida que la sociedad entiende la gravedad del problema y se activan normativas como la Ley Orgánica de medidas de protección integral contra la violencia de género (Jefatura del Estado, 2004). En este periodo solo un 24,5\% de las canciones fomentaban la violencia de género frente a un 53,6 \% que la denunciaban contribuyendo a una mayor distribución de estos mensajes entre los adolescentes. Esta tendencia a favorecer la distribución de contenidos musicales que denuncian la violencia de género cambia significativamente en la actualidad (2010 - 2018), donde se puede observar un aumento preocupante de la presencia de canciones que fomentan la violencia de género $(34,7 \%)$ frente a las que la denuncian $(26,9 \%)$. Aumenta también la distribución masiva de contenidos que fomentan la violencia $(23,5 \%)$ frente a los que la denuncian (5,5\%). Esto lleva a concluir que el peso relativo de la música que promociona la violencia física o simbólica hacia la mujer es mucho mayor en la actualidad y su mensaje está más presente en la sociedad, lo que puede favorecer cierta familiarización con este tipo de discursos.

Por otra parte, el análisis sobre cómo están distribuidos los discursos que fomentan o denuncian la violencia de género en los estilos musicales más consumidos por la gente joven arroja datos muy relevantes (Tabla 2). 


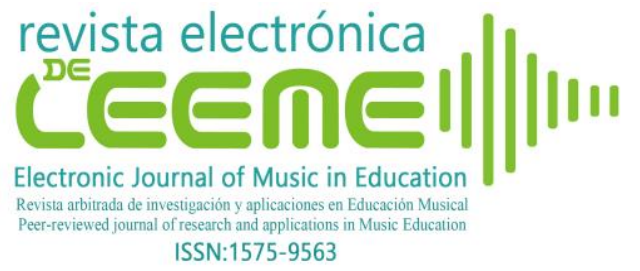

Tabla 2. Violencia de género por estilos musicales. Porcentajes $(n=350)$

\begin{tabular}{lcc}
\hline Estilo musical & Fomenta & Denuncia \\
\hline & 36,7 & 33,0 \\
Pop & 8,2 & 17,5 \\
Rock & 5,1 & 17,5 \\
Heavy Metal & 5,1 & 13,1 \\
Punk & 2,1 & 10,7 \\
Hip Hop & 1,0 & 2,4 \\
Flamenco & 9,2 & 2,8 \\
Canción española & 26,5 & 1,9 \\
Reggaetón & 6,1 & 1,1 \\
Otros &
\end{tabular}

Fuente: elaboración propia

Los discursos que denuncian la violencia se encuentran más presenten en géneros musicales como el rock $(17,5 \%)$, heavy metal $(17,5 \%)$, punk $(13,1 \%)$ y hip hop $(10,7 \%)$. Lo preocupante aparece cuando se analizan los estilos que favorecen la distribución de mensajes que fomentan la violencia de género y que están muy relacionados con las pautas de ocio que experimenta actualmente la juventud como el pop $(36,7 \%)$, o el reggaetón (26,5 \%). Llama también la atención que un género musical como el de la canción española, pese a no tener un peso muy importante en la muestra, tenga un porcentaje significativo de temas que fomentan la violencia $(9,2 \%)$. Este género musical no es el que más se distribuye entre los jóvenes, pero es un género musical consumido por generaciones anteriores (padres y abuelos) y también contribuye a consolidar su discurso musical. La música que se escucha en los ámbitos de la vida cotidiana (en casa, en el coche durante un viaje en familia, las fiestas familiares, etc.) influye en el proceso de socialización del alumnado adolescente, ayudándole a construir una memoria musical que le familiariza con un lenguaje que normaliza algunas pautas de violencia de género (North, Hargreaves y O’Neil, 2000).

Por otro lado, tal y como muestra la Tabla 3, actualmente la mayoría de las canciones que denuncia la violencia de género $(19,4 \%)$ lo hace empoderando en sus textos a la mujer $(15,4 \%)$ y ofreciendo alguna alternativa de acción para salir de esa situación de agresión.

Tabla 3. Denuncia de violencia de género, empoderamiento de la mujer $(n=350)$

\begin{tabular}{cccc}
\hline Año & Denuncia \% & Empoderamiento \% & \% Sobre el total \\
\hline Antes 1980 & 2,0 & 1,8 & 3,8 \\
$1980-1989$ & 3,7 & 2,9 & 8,3 \\
$1990-1999$ & 8,3 & 4,8 & 13,4 \\
$2000-2009$ & 38,6 & 28,6 & 45,4 \\
$2010-2018$ & 19,4 & 15,4 & 29,1 \\
\hline Total & 72,0 & 53,4 & 100 \\
\hline \multicolumn{4}{c}{ Fuente: elaboración propia }
\end{tabular}

@ Jaime Hormigos Ruiz. The content of this article is the sole responsibility of the authors. The Revista Electrónica de LEEME and Universitat de València are not liable for any legal actions that may arise involving the article's content. Revista Electrónica de LEEME - Lista Electrónica Europea de Música en la Educaciónhttp://ojs.uv.es/index/php/LEEME/index ISSN: 1575-9563. Editores: Universidad de Valencia y Jesús Tejada. Visibilidad de esta revista: SCOPUS, Emerging Sources Citation Index (Clarivate), EBSCO, CINDOC (CSIC), Citefactor, COPAC, Dialnet, DICE (CSIC), DOAJ, e-revistas (CSIC), EBSCO Premier, ERIH+, Gale Cengage Learning, INRECS, IRESIE, LATINDEX, MIAR, OCLC Worldcat, RESH, REDIB, RILM Core Journals, SUDOC, ULRICHS. Esta revista es de acceso libre mediante licencia Creative Commons 4.0 CC by. Política de archivado: etiqueta verde SHERPA-ROMEO. 


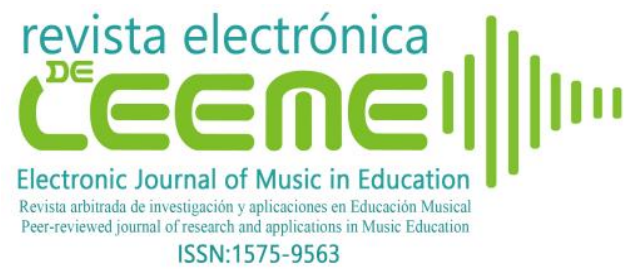

En este sentido, se puede observar una evolución importante. En la década 1990 - 1999 un $13,4 \%$ de todas las canciones analizadas trataban la violencia de género, de las cuales solo un 4,8\% denunciaba la situación potenciando un mensaje de empoderamiento de la mujer. El resto de canciones que denunciaban el tema en esta época $(13,4 \%)$ lo hacían únicamente describiéndolo. Durante ese periodo, tenían más éxito los discursos que favorecían las actitudes violentas, enmascarando estos comportamientos en procesos de interacción habituales en las relaciones de pareja. Esta normalización de las actitudes violentas contribuía a que no se visualizara socialmente a la víctima ni al problema. De este modo, canciones de mucho éxito en esa época contribuyeron a normalizar pautas de violencia de género (Berrocal y Gutiérrez, 2002).

En el periodo 2000 - 2009, la situación cambia significativamente. El problema de la violencia de género se visibiliza con mayor facilidad en el ámbito social y esto se empieza a ver reflejado también en las manifestaciones culturales. Así, se puede observar que el $45 \%$ del total de canciones analizadas en la muestra se sitúan en este periodo, denunciando la violencia de género $\mathrm{y}$, de estas, un $28,6 \%$ denuncian la situación lanzando un mensaje directo hacia las víctimas y la sociedad que contribuye activamente al empoderamiento de la mujer y a la transmisión de un discurso educativo que facilita la comprensión del problema.

Esta tendencia continúa en la actualidad, donde se puede observar que el 29,1\% del total de temas que integran la muestra tratan directamente la violencia de género, y de este porcentaje de canciones, el 15,4 \% lo hace a través de una función educativa constante que favorece el empoderamiento de la mujer.

\section{Propuesta metodológica de trabajo en el aula}

El alumnado actual se caracteriza por ser más interactivo, espontáneo, crítico y dinámico en el uso de las nuevas tecnologías. Esto provoca que demande al sistema educativo experiencias y sensaciones nuevas que capten su atención y potencien el conocimiento activo. Apostar por el desarrollo de técnicas innovadoras de enseñanza y aprendizaje es una necesidad constante para el docente. Diversos modelos de pedagogía activa como los de Paynter (1982), Schafer (2004) o Gardner (2010) utilizan la música como material educativo que ofrece una oportunidad excepcional para conectar con la realidad social. Las canciones se convierten en un potente agente socializador gracias al cual las nuevas generaciones pueden aprender valores y desarrollar un pensamiento crítico hacia determinados problemas sociales. En este sentido, se propone un ejercicio de pedagogía activa para trabajar con adolescentes el problema de la violencia de género a través de la música que habitualmente escuchan. La actividad se diseña para el trabajo con estudiantes de ambos sexos que cursan Educación Secundaria Obligatoria y Bachillerato, y está pensada para trabajar las materias de música, cultura audiovisual y valores éticos.

@ Jaime Hormigos Ruiz. The content of this article is the sole responsibility of the authors. The Revista Electrónica de LEEME and Universitat de València are not liable for any legal actions that may arise involving the article's content. Revista Electrónica de LEEME - Lista Electrónica Europea de Música en la Educación-. http://ojs.uv.es/index/php/LEEME/index ISSN: 1575-9563. Editores: Universidad de Valencia y Jesús Tejada. Visibilidad de esta revista: SCOPUS, Emerging Sources Citation Index (Clarivate), EBSCO, CINDOC (CSIC), Citefactor, COPAC, Dialnet, DICE (CSIC), DOAJ, e-revistas (CSIC), EBSCO Premier, ERIH+, Gale Cengage Learning, INRECS, IRESIE, LATINDEX, MIAR, OCLC Worldcat, RESH, REDIB, RILM Core Journals, SUDOC, ULRICHS. Esta revista es de acceso libre mediante licencia Creative Commons 4.0 CC by. Política de archivado: etiqueta verde SHERPA-ROMEO. 


\subsection{Justificación de la actividad}

Los temas transversales en el ámbito educativo ofrecen al alumnado la posibilidad de adquirir valores vinculados a la realidad social. En el caso de la lucha por erradicar la violencia de género entre el alumnado adolescente, es fundamental introducir en el currículo de los centros educativos un tipo de enseñanza que responda a este problema social y que tenga presente la formación de valores que ayude a desarrollar capacidades de comprensión y actuación social (Díaz, 2006). En esta propuesta, se busca trabajar la violencia de género como un tema educativo transversal. De este modo, siguiendo el método planteado por Flores (2007) o Valls, Puigvert y Duque (2008) se propone una actividad que permita:

1. Analizar el problema de la violencia de género.

2. Ayudar a entender las variables sociológicas del problema.

3. Identificar estas variables en el discurso de la música que rodea al adolescente.

4. Utilizar la música popular para transmitir valores positivos que ayuden a combatir el problema.

Para desarrollar esta metodología educativa se utilizarán canciones que formen parte del oído social del adolescente y que visibilicen el problema de la violencia de género empoderando el papel de la mujer en la sociedad. De este modo, se conseguirá llamar la atención del joven hacia contenidos culturales que forman parte de su día a día facilitando su compromiso participativo y despertando su interés en el tema. Analizar la violencia de género a través de las canciones en el aula permitirá trabajar, por un lado, con un medio de información directa que ayuda a visibilizar el problema y, al mismo tiempo, con un escenario simbólico y cultural que ayuda a establecer propuestas de intervención que quedan fijadas con fuerza en el universo simbólico del adolescente (Hall, 2014).

\subsection{Objetivos de la actividad}

Mediante el desarrollo de esta actividad se conseguirán cinco objetivos fundamentales. En primer lugar (O1), fomentar un análisis crítico del texto de la canción que favorezca el aprendizaje significativo y potencie la transmisión de valores socialmente aceptados, reforzando comportamientos positivos frente a la violencia de género en la sociedad en general y en la juventud en particular. En segundo lugar (O2), fomentar una actitud crítica frente a los discursos que favorecen la violencia de género en los contenidos culturales, en general, y en la música en particular. En tercer lugar (O3), trabajar la acción comunicativa de la canción practicando la escucha activa. Como cuarto objetivo (O4), trabajar con las actitudes interiores del estudiante para ayudarle a combatir prejuicios y fomentar la reflexión sobre la violencia de género como

@ Jaime Hormigos Ruiz. The content of this article is the sole responsibility of the authors. The Revista Electrónica de LEEME and Universitat de València are not liable for any legal actions that may arise involving the article's content. Revista Electrónica de LEEME - Lista Electrónica Europea de Música en la Educación-. http://ojs.uv.es/index/php/LEEME/index ISSN: 1575-9563. Editores: Universidad de Valencia y Jesús Tejada. Visibilidad de esta revista: SCOPUS, Emerging Sources Citation Index (Clarivate), EBSCO, CINDOC (CSIC), Citefactor, COPAC, Dialnet, DICE (CSIC), DOAJ, e-revistas (CSIC), EBSCO Premier, ERIH+, Gale Cengage Learning, INRECS, IRESIE, LATINDEX, MIAR, OCLC Worldcat, RESH, REDIB, RILM Core Journals, SUDOC, ULRICHS. Esta revista es de acceso libre mediante licencia Creative Commons 4.0 CC by. Política de archivado: etiqueta verde SHERPA-ROMEO. 


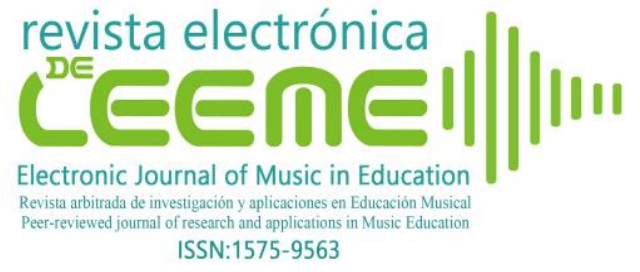

problema social. Por último (O5), plantear respuestas efectivas en la resolución del problema de la violencia de género en la sociedad.

\subsection{Ejercicio}

El método que aquí se plantea consiste en la escucha dirigida y controlada de canciones que tratan el tema de la violencia de género. De este modo, se propone trabajar este tipo de violencia en el aula a través de un ejercicio dividido en tres partes: a) Escucha activa y guiada, b) Análisis: trabajando con los objetivos $(\mathrm{O})$ y c) Debate final.

\subsubsection{Escucha activa y guiada}

El ejercicio comienza planteando una conversación de encuadre donde el educador explicará al estudiantado la importancia de la violencia de género como problema social y les mostrará cómo se puede detectar pautas de violencia de género en las canciones de música popular más de moda en su sociedad. Posteriormente, se pasará a realizar la escucha de 5 canciones que traten el problema. La selección de los temas se deberá hacer en función de las siguientes variables:

a) Estilo musical: se seleccionarán canciones que se ajusten, lo máximo posible, a los géneros musicales cercanos a los adolescentes ya que este criterio provoca más simpatías o antipatías hacia el tema que se está escuchando. Seleccionar correctamente las canciones que se ajusten al gusto social de los estudiantes favorecerá la identificación de las características sociales presentes en el tema, el conocimiento de las reglas de expresión y del lenguaje utilizado y activará el comportamiento emocional. Se escogerán estilos musicales muy distribuidos y con intérpretes conocidos.

b) Letra de la canción: la letra es crucial para fijar el mensaje con fuerza. Deberá sintetizar una idea clara y directa a modo de eslogan (Negus y Astor, 2015). La letra debe ser en castellano para favorecer su comprensión, fácil de recordar, con un mensaje directo y acompañada de una música pegadiza.

c) Contenido denotativo de la canción: se seleccionarán canciones que traten directamente la violencia de género desde distintas perspectivas para abrir al máximo el debate.

Teniendo en cuenta estas variables, se propone analizar el problema a través de las siguientes 5 canciones:

\footnotetext{
@ Jaime Hormigos Ruiz. The content of this article is the sole responsibility of the authors. The Revista Electrónica de LEEME and Universitat de València are not liable for any legal actions that may arise involving the article's content. Revista Electrónica de LEEME - Lista Electrónica Europea de Música en la Educaciónhttp://ojs.uv.es/index/php/LEEME/index ISSN: 1575-9563. Editores: Universidad de Valencia y Jesús Tejada. Visibilidad de esta revista: SCOPUS, Emerging Sources Citation Index (Clarivate), EBSCO, CINDOC (CSIC), Citefactor, COPAC, Dialnet, DICE (CSIC), DOAJ, e-revistas (CSIC), EBSCO Premier, ERIH+, Gale Cengage Learning, INRECS, IRESIE, LATINDEX, MIAR, OCLC Worldcat, RESH, REDIB, RILM Core Journals, SUDOC, ULRICHS. Esta revista es de acceso libre mediante licencia Creative Commons 4.0 CC by. Política de archivado: etiqueta verde SHERPA-ROMEO.
} 
Tabla 4. Canciones para analizar la violencia de género en el aula

\begin{tabular}{llccl}
\hline Canción/Título & Intérprete & Estilo & Año & Objetivo trabajado \\
\hline Las que faltaron & Mafalda & Rock & 2018 & O1, O2, O3 \\
\hline La puerta violeta & Rozalén & Pop & 2017 & O1, O3, O5 \\
\hline La bella y la bestia & Porta & Rap & 2009 & O1, O2, O3, O5 \\
\hline Cobarde & Warcry & Metal & 2011 & O1, O3, O4, O5 \\
\hline Lo malo & Ana Guerra & Pop & 2019 & O3, O4, O5 \\
\hline
\end{tabular}

Fuente: elaboración propia

\subsubsection{Análisis}

En primer lugar, el análisis comienza enseñando a los estudiantes el importante papel de la mujer en el desarrollo de la sociedad moderna. Para ello, se utilizará la canción Las que faltaron (Mafalda, 2018). Utilizando un ritmo rock y un lenguaje muy directo, se centrará el análisis en resaltar la presencia de la mujer en la historia, haciendo hincapié en las siguientes partes de la letra para trabajar los objetivos $\mathrm{O} 1, \mathrm{O} 2, \mathrm{O} 3$ :

\footnotetext{
"En el arte, la ciencia, el deporte / siempre hay una constante (...) miro atrás y no están (...) las que siempre faltarán / mujeres que hicieron historia / mujeres murieron sin gloria / buscas referentes en libros / en la escuela no tienen memoria".

"Aganice de Tesalia la llamaron bruja por predecir eclipses, por predecir la luna / Ada Lovelace, matemática y visionaria trabajó con los números y los puso en una máquina/ Lynn Margulis habló sobre la evolución / Chin Shin fue una pirata que comandó una legión / Pamela Lyndon Travers voló en paraguas/ contra griegos, romanos y egipcios estaba Cleopatra / J.K. Rowling se llama Joanne pero vendió más al esconder su nombre real (...)/ Marsha P. Johnson era trans y negra y luchó por sus derechos, ella siempre estuvo en guerra (...)/ Julia Hermosilla, casi nos libra de Franco en dos ocasiones (...) / Dolores Ibárruri gritó "No pasarán" / Nina Simone, Etta James, Billie Holiday, Janis Joplin, Edith Piaf hoy me ayudan a cantar / Violeta Parra, Mercedes Sosa, Chavela Vargas /con ternura y rabia en la garganta".
}

En segundo lugar, se analiza el problema de la violencia de género de forma directa. Para ello, se utilizarán dos canciones, La puerta violeta (Rozalén, 2017) y La bella y la bestia (Porta, 2009). En este caso se recurre al pop y el rap para analizar el tema. El pop facilitará poder trabajar con estribillos pegadizos que ayuden a fijar los conceptos y el rap a introducir un lenguaje más elaborado que amplíe el vocabulario de los estudiantes. En esta parte del análisis, se resalta en primer lugar la angustia que supone para alguien que sufre malos tratos enfrentarse a esa situación, trabajando los objetivos $\mathrm{O} 1$ y O3:

\footnotetext{
"Una niña triste en el espejo me mira prudente y no quiere hablar / Hay un monstruo gris en la cocina que lo rompe todo que no para de gritar / Tengo una mano en el cuello que con sutileza me impide respirar / Una venda me tapa los ojos puedo oler el miedo y se acerca (...) / Tengo todo el cuerpo encadenado / Las manos agrietadas mil arrugas en la piel / Las fantasmas hablan en la nuca / Se reabre la herida y me sangra".
}

Se utilizará otra parte de la canción para dar un mensaje positivo sobre los beneficios de combatir la violencia de género ayudando a la víctima a salir de esta situación (O5): 
"Pero dibujé una puerta violeta en la pared / Y al entrar me liberé como se despliega la vela de un barco / Desperté en un prado verde muy lejos de aquí / Corrí, grité, reí / Sé lo que no quiero / Ahora estoy a salvo".

Una vez mostradas las consecuencias que la violencia de género provoca en la víctima, se desarrollará más el análisis describiendo el ciclo de la violencia de género: "inicio de la relación", "agresión", "luna de miel", "desenlace". Para ello, servirá la letra de La bella y la bestia (Porta, 2009) que ayudará a trabajar los objetivos O1, O2, O3, O5. Se deberá hacer especial hincapié en las siguientes partes de la letra:

\begin{abstract}
"Todo marchaba bien / Eso parecía en su primera luna de miel / Juró serle de por vida fiel y ella a él / Una historia como otra cualquiera / Quién les ve y quién les viera (...) / Pero el tiempo pasa y las relaciones se agotan, se cansan / Ella ni lo nota porque esta ciega de amor / Pero él no aguanta la monotonía, ya no quería ser dueño de una sola tía (...) / La primera vez fue la más dolorosa / Te regaló una infidelidad por cada rosa / Y es que el perdón será tu debilidad / Pero lo que pasa una vez siempre sucede una vez más".

"Empiezan las discusiones / Parece que a él no le gustan / Se vuelve insensible y agresivo y a bella le asusta / Lágrimas caían, tras un empujón y el primer puñetazo / Te conformas con un perdón y un simple abrazo (...) / No quieres darle importancia porque no quieres perderlo / Pero sientes impotencia y a la vez pánico y miedo (...) / El silencio no te ayuda / Sé que no sabes que hacer / Sabes que fue la primera y no será la última vez / Créeme sé que no quieres más problemas pero no te quedes en silencio si tu marido te pega / Porque no le perteneces / Te mereces mucho más (...) / Bestia no te quiere pero quiere que seas suya para siempre $/{ }_{i} \mathrm{Si}$ no eres mía, no serás de nadie, entiendes! / Cuando ella quiso hablar ya era demasiado tarde".
\end{abstract}

En tercer lugar, el análisis se centrará en la posición que el maltratador tiene en la sociedad. Hay comportamientos que suponen violencia de género pero que la sociedad acepta, bien porque están normalizados o bien porque se consideran problema de las personas que mantienen la relación. Enseñar al adolescente a estigmatizar a quien lleva a cabo estos comportamientos puede ayudar a la hora de trabajar su respuesta ante la violencia de género. Para este fin se utiliza la canción Cobarde (Warcry, 2011). En esta ocasión se elige un estilo metal que permitirá hacer llegar el mensaje de una forma más directa y con mucha más fuerza. En este tema se trabajarán los objetivos $\mathrm{O} 1, \mathrm{O} 3, \mathrm{O} 4$ y O5 centrando el análisis en la siguiente parte de la letra:

\footnotetext{
“¿Quién lo sabía? ¿Quién lo pensó? / Nada en sus gestos lo delató / Nadie la oía, nadie la vio girar la cara a su dolor / Soñó lo feliz que era con él / Lloraba a escondidas intentando callar la verdad / Maldigo al que pegue a una mujer / Que el infierno se cebe con él / Cobarde... cobarde... cobarde".
}

Por último, el ejercicio se centra en trabajar un mensaje de empoderamiento de la mujer analizando los cambios que se están produciendo en las relaciones entre jóvenes a medida que la sociedad va siendo más consciente del grave problema que supone la violencia de género. Para ello se trabajará con la canción Lo malo (Guerra, 2018). Se recurre de nuevo a un tema pop de mucho éxito en la sociedad española para fijar la idea que se quiere transmitir a través del estribillo pegadizo. En este sentido se trabajan los objetivos O3, O4 y O5 escuchando activamente la siguiente parte de la letra: 


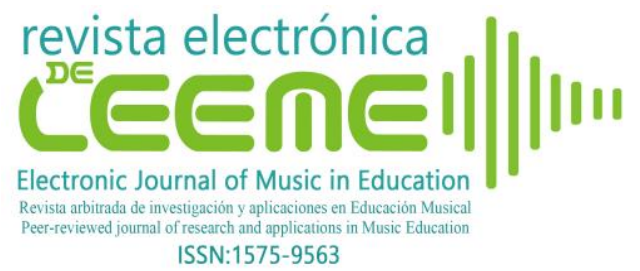

"Yo decido el cuándo, el dónde y con quién / Que voy a darme a mí de una y otra vez / Lo que tanto me quité, que pa' ti tan poco fue (...) / Tengo claro que no me voy a fijar en un chico malo no, no, no / Pa' fuera lo malo no, no, no / Yo no quiero nada malo no, no, no / En mi vida malo no, no, no / Tú ya no estás dentro de mí / Se han podrido las flores aquí / Ahora ya no quiero rosas / Soy el león que se comió las mariposas (...) / Esta noche bailo mejor sin ti (...) / Yo no te miro, y tú me vas a ver / Yo no te escucho, y tú me vas a oír / Paso de largo y paso de ti / Esta noche bailo solo para mí".

\subsubsection{Debate final}

Por medio del debate final se pretende abrir el aprendizaje académico y facilitar el intercambio de opiniones entre los estudiantes sobre el tema que se está analizando con el fin de facilitar la conexión entre el contenido trabajado en clase y su vida cotidiana. En este sentido, el debate ayudará al estudiante a identificar las señales sociales que explican el problema de la violencia de género, conocer el lenguaje y reglas de expresión que se utilizan para definirle y trabajar sus emociones en un entorno grupal.

El trabajo de escucha activa y dirigida propuesto cumple una serie de funciones básicas dentro de todo proceso educativo que se verá reflejado en el debate: favorece la comunicación y la expresión emocional del estudiante (Ciurana y Alsina, 2019), favorece la representación simbólica de un problema y, por último, favorece la atención a través del entretenimiento, fomentando la comprensión. En este sentido, la audición guiada crea un discurso basado en la semántica de racimo (Luna, 2008) que proporciona una comunicación desde el intercambio de opiniones, creencias, conocimientos, información, sentimientos o emociones. El debate final servirá al alumnado para expresar lo que piensa y siente sobre el tema analizado de manera que otros puedan recogerlo y actuar en consecuencia.

\section{Conclusiones}

A través del desarrollo de esta investigación se ha constatado que las canciones que denuncian la violencia de género son más numerosas en la sociedad española (72\% de la muestra analizada), frente a aquellos otros temas que la fomentan (28\%). A pesar de esto, el discurso que fomenta la violencia tiene mayor distribución entre los adolescentes porque los estilos en los que se encuentran incluidos esos temas están más de moda. Si la música más distribuida por los medios de comunicación es aquella que trasmite valores que pueden fomentar la violencia hacia la mujer, parte de esos comportamientos se entenderán como válidos en los procesos de interacción de la juventud. Para evitar esto, es conveniente potenciar la escucha activa de aquellas canciones que denuncian la violencia y favorecen la igualdad de roles entre hombre y mujer. En este sentido, la interacción en el proceso educativo entre el lenguaje verbal y el musical que se propone en este trabajo facilitará esa escucha activa y permitirá

@ Jaime Hormigos Ruiz. The content of this article is the sole responsibility of the authors. The Revista Electrónica de LEEME and Universitat de València are not liable for any legal actions that may arise involving the article's content. Revista Electrónica de LEEME - Lista Electrónica Europea de Música en la Educación-. http://ojs.uv.es/index/php/LEEME/index ISSN: 1575-9563. Editores: Universidad de Valencia y Jesús Tejada. Visibilidad de esta revista: SCOPUS, Emerging Sources Citation Index (Clarivate), EBSCO, CINDOC (CSIC), Citefactor, COPAC, Dialnet, DICE (CSIC), DOAJ, e-revistas (CSIC), EBSCO Premier, ERIH+, Gale Cengage Learning, INRECS, IRESIE, LATINDEX, MIAR, OCLC Worldcat, RESH, REDIB, RILM Core Journals, SUDOC, ULRICHS. Esta revista es de acceso libre mediante licencia Creative Commons 4.0 CC by. Política de archivado: etiqueta verde SHERPA-ROMEO. 


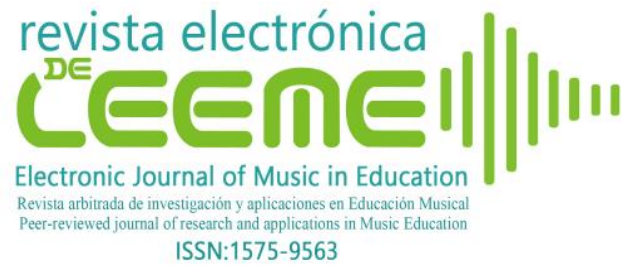

utilizar las canciones como una potente herramienta educativa para analizar y comprender el problema de la violencia de género.

\section{Referencias}

Guerra, A. (2018). Lo malo. Reflexión [CD]. Madrid: Universal. Recuperado de: https://www.youtube.com/watch?v=qYXCsZunjRM

Berrocal, E. y Gutiérrez, J. (2002). Música y género: análisis de una muestra de canciones populares. Comunicar, 18, 187-190. doi:10.3916/C18-2002-30

Berrón, E. (2017). Integración creativa de la música Pop-Rock en el aula. Revista Electrónica de LEEME, 39, 1-20. doi:10.7203/LEEME.39.10070

Branch, A. (2012). All the young dudes: educational capital, masculinity and the uses of popular music. Popular Music, 31(1), 25-44. doi:10.1017/50261143011000444

Ciurana, M. y Alsina, M. (2019). El aula de música como espacio sonoro de aprendizaje. Revista Electrónica de LEEME, 44, 42-62. doi:10.7203/LEEME.44.15595

Díaz, A. (2006). La educación en valores: Avatares del currículum formal, oculto y los temas transversales. Revista Electrónica de Investigación Educativa, 8(1). Recuperado de: https://redie.uabc.mx/redie/article/view/117/201

FGEE (2019). Barómetro de hábitos de lectura y compra de libros de 2018. Madrid: Federación de Gremios de Editores de España y Ministerio de Cultura y Deporte.

Flores, S. (2007). Principales acercamientos al uso de la música popular actual en la Educación Secundaria. Revista Electrónica de LEEME, 19, 1-16. Recuperado de: https://ojs.uv.es/index.php/LEEME/article/view/9763/9197

García, R., Ruiz, E. y Rebollo, A. (2016). Preferencias relacionales de género en el contexto escolar: Una nueva medida para el diagnóstico de relaciones de género en educación. RELIEVE, 22(1), 1-22. doi:10.7203/relieve22.1.6877

Gardner, H. (2010). La inteligencia reformulada: las inteligencias múltiples en el siglo XXI. Barcelona: Paidós.

Hormigos, J., Gómez, M. y Perelló, S. (2018). Música y violencia de género en España. Estudio comparado por estilos musicales. Convergencia Revista de Ciencias Sociales, 76, 7598. doi:10.29101/crcs.v25i76.4291 
Gómez, M., Hormigos, J. y Perelló, S. (2019). El ciclo de la violencia contra las mujeres en las canciones de música popular en España. Andamios. Revista de Investigación Social, 16(41), 331-353. doi:10.29092/uacm.v16i41.728

Hall, R. (2014). Enhancing the popular music ensemble workshop and maximising student potential through the integration of creativity. International Journal of Music Education, 33(1), 103-112. doi:10.1177/0255761414533310

Hennion, A. (2010). Gustos musicales: De una sociología de la mediación a una pragmática del gusto. Comunicar, 34, 25-33. doi:10.3916/C34-2010-02-02

Herrera, L., Cremades, R. y Lorenzo, O. (2010). Preferencias musicales de los estudiantes de educación secundaria obligatoria: influencia de la educación formal e informal. Cultura y educación, 22, 37-51. doi:10.1174/113564010790935222

IFPI (2006-2019). Global Music Report. Bruselas: International Federation of the Phonographic Industry. Recuperado de: http://www.ifpi.org

INE (2020). Estadística de Violencia Doméstica y de Género 2019. Madrid: Instituto Nacional de Estadística.

Jefatura del Estado (2004). Ley Orgánica 1/2004, de 28 de diciembre, de Medidas de Protección Integral contra la Violencia de Género. BOE (28/12/2004), n³13, referencia 21760, pp.42166-422197.

Karvelis, N. (2018). Race, Class, Gender, and Rhymes: Hip-Hop as Critical Pedagogy. Music Educators Journal, 105(1), 46-50. doi:10.1177/0027432118788138

Kelly, S. (2002). A Sociological Basis for Music Education. International Journal of Music Education, 39(1), 40-49. doi:10.1177/025576140203900105

Kratus, J. (2017). Music Listening is Creative. Music Educators Journal, 103(3), 46-51. doi: $10.1177 / 0027432116686843$

Lorente, M. (2007). Violencia de género, educación y socialización: acciones y reacciones. Revista de Educación, 342, 19-35. doi:10.4438/1988-592X-0034-8082-RE

Luna, M. (2008). La enseñanza audiovisual para el fomento de competencias múltiples. Comunicar, 31, 469-475. doi:10.3916/c31-2008-03-037

Mafalda (2018). Las que faltaron. En Palabras forman caos [CD]. Valencia, Esp.: Gazpatxo Fest Cultura. Recuperado de: https://www.youtube.com/watch?v=ji5CVZczEks

@ Jaime Hormigos Ruiz. The content of this article is the sole responsibility of the authors. The Revista Electrónica de LEEME and Universitat de València are not liable for any legal actions that may arise involving the article's content. Revista Electrónica de LEEME - Lista Electrónica Europea de Música en la Educaciónhttp://ojs.uv.es/index/php/LEEME/index ISSN: 1575-9563. Editores: Universidad de Valencia y Jesús Tejada. Visibilidad de esta revista: SCOPUS, Emerging Sources Citation Index (Clarivate), EBSCO, CINDOC (CSIC), Citefactor, COPAC, Dialnet, DICE (CSIC), DOAJ, e-revistas (CSIC), EBSCO Premier, ERIH+, Gale Cengage Learning, INRECS, IRESIE, LATINDEX, MIAR, OCLC Worldcat, RESH, REDIB, RILM Core Journals, SUDOC, ULRICHS. Esta revista es de acceso libre mediante licencia Creative Commons 4.0 CC by. Política de archivado: etiqueta verde SHERPA-ROMEO. 
Negus, K. y Astor, P. (2015). Songwriters and song lyrics: architecture, ambiguity and repetition. Popular Music, 34 (2), 226-244. doi:10.1017/S0261143015000021

North, A. C., Hargreaves, D. J. y O`Neil, S. A. (2000). The importance of music to adolescents. British Journal of Educational Psychology, 70, 255-272. doi:10.1348/000709900158083

Padrós, M., Aubert, A. y Melgar, P. (2010). Modelos de atracción de los y las adolescentes. Contribuciones desde la socialización preventiva de la violencia de género. Pedagogía Social: revista interuniversitaria, 17, 73 - 82. doi:10.7179/PSRI_2010.17.06

Paynter, J. (1982). Music in the Secondary School Curriculum. Cambridge: Cambridge University Press.

Perelló-Oliver, S. (2009). Metodología de la investigación social. Madrid: Dykinson.

Porta (2009). La bella y la bestia. Trastorno bipolar [CD]. Madrid: Universal. Recuperado de: https://www.youtube.com/watch?v=woP0xqH_5rA

Prendes, M., García, P. y Solano, I. (2020). Igualdad de género y TIC en contextos educativos formales: Una revisión sistemática. Comunicar, 63, 9-20. doi:10.3916/C63-2020-01

Promusicae (2003-2019). Top canciones más distribuidas en España por año. Madrid: Productores de música de España. Recuperado de: www.promusicae.es

Rozalén (2017). La puerta violeta. Cerrando puntos suspensivos [CD]. Madrid: Sony: Recuperado de: https://www.youtube.com/watch?v=gYyKuLV8A_c

Schafer, R. M. (2004). El rinoceronte en el aula. Buenos Aires: Ricordi.

SGAE (1999-2019). Anuarios de las artes escénicas, musicales y audiovisuales. Madrid: Sociedad General de Autores y Editores. Recuperado de: http://www.anuariossgae.com

Valls, R., Puigvert, L. y Duque, E. (2008). Gender violence among teenagers socialization and prevention. Violence Against Women, 14(7), 759-785. doi:10.1177/1077801208320365

Warcry (2011). Cobarde. Alfa [CD]. Oviedo: Jaus Records. Recuperado de: https://www.youtube.com/watch?v=FH8fM1nuL8U

@ Jaime Hormigos Ruiz. The content of this article is the sole responsibility of the authors. The Revista Electrónica de LEEME and Universitat de València are not liable for any legal actions that may arise involving the article's content. Revista Electrónica de LEEME - Lista Electrónica Europea de Música en la Educaciónhttp://ojs.uv.es/index/php/LEEME/index ISSN: 1575-9563. Editores: Universidad de Valencia y Jesús Tejada. Visibilidad de esta revista: SCOPUS, Emerging Sources Citation Index (Clarivate), EBSCO, CINDOC (CSIC), Citefactor, COPAC, Dialnet, DICE (CSIC), DOAJ, e-revistas (CSIC), EBSCO Premier, ERIH+, Gale Cengage Learning, INRECS, IRESIE, LATINDEX, MIAR, OCLC Worldcat, RESH, REDIB, RILM Core Journals, SUDOC, ULRICHS. Esta revista es de acceso libre mediante licencia Creative Commons 4.0 CC by. Política de archivado: etiqueta verde SHERPA-ROMEO. 